\title{
A REVIEW ON MECHANICAL PROPERTIES OF INTRA-LAYER
}

\section{PALM FIBER (PALMYRA SPOUT) - FLAX - S-GLASS \\ FIBER REINFORCED COMPOSITE}

\section{PRABU, T. BABU, K. PRADEEP \& S. SANJAYARUN}

Faculty of Mechanical Engineering, Sri Sai Ram Engineering College, West-Tambaram, Chennai, Tamil Nadu, India

Normally natural fibers are extracted from natural sources like plants and animals. The composite materials made by natural fibers plays the wide range of applications in engineering fields because of eco-friendly and low cost. The aim of this review article is to provide the comprehensive review of the mechanical properties and their applications of natural fiber reinforced composite using palm (Palmyra spout), flax fibers along with S-glass fibers. In addition, it summarises various surface treatments applied to natural fibers and their effect on natural fiber reinforced composite properties. The fabrication is done by injection molding method. The various mechanical property tests (tensile test, hardness test, impact test etc...) are to be conducted over the fabricated composite samples through references listed for better results and applications. It has been concluded that the S-glass fiber coating over the surfaces of hybrid fiber improves the mechanical properties tremendously and the chemical treatment of the natural fiber improves adhesion between the fibers. The hybrid fiber coated with glass fiber in this composite plays an important role in determining the mechanical properties.
\end{abstract}

KEYWORDS: Natural Palm and Flax Fibres, S-Glass Fibres, Composite Fabrication, Injection Moulding \& Mechanical Properties

Received: Mar 30, 2018; Accepted: Apr 19, 2018; Published: May 14, 2018; Paper Id.: IJMPERDJUN201853

\section{INTRODUCTION}

In recent days the synthetic fibers are replaced by natural fibers because of low cost, less weight, low density, high specific strength and eco-friendly nature. Thermoplastic or thermosetting plastics is been used as resin mixed up with respective hardener in synthetic fiber composites. The applications of synthetic fiber composites are very few due to the high cost of materials and manufacturing methods [1] [3].

Composites are heterogeneous in nature, produced by combining two or more components with reinforcing fibers and compactable matrix. Composite materials are replacing the existing conventional materials due to their superior properties, such as high strength-to-weight ratio, high mechanical strength and minimum thermal expansion. Generally, the natural fibers are extracted naturally from plant and animals in the elongated form. The various plant fibers are cotton, hemp, jute, sisal, pineapple, ramie, bamboo, banana, etc.., as well as wood and seeds of flax are used as the reinforcement in polymer matrix composites.[2] [4].

The application of natural fiber reinforced composites exists many possibilities since the number of applications is rapidly growing within many engineering fields. The main purpose of using composite in recent years is lighter in weight for constructional purpose and more vibration resistant in structures. 
Through various research and experimental investigation on natural fiber composites, it's been observed as, it reduces the weight of the existing component by 10-28\% considerably and also it gives good improved material properties results (tensile, hardness, stiffness, thermal expansion etc...) compared with the existing ones [8]. The NFCs material plays important roles in automobile industries, daily life applications, aerospace body parts manufacturing, etc. The NFCs replaces the existing one because of easy availability of source material and low cost, it can be recycled, low specific weight, it does not harmful to the environment conditions, good corrosion resistance, thermal retardance, and it consumes low energy compared with the synthetic composites and conventional materials. The tensile strength of composite materials is four to six times greater than that of the conventional engineering materials. [5].

\section{Application of NFCs}

The applications of the natural fiber composites are not limited to particular fields, in recent technological development, the NFCs gives a wide range of applications such as automotive interior and exterior parts, aircraft machine parts, electrical appliances and daily life household appliances etc... [9] [10]. The leading automotive industries uses the natural fiber composite materials because, the overall weight of the vehicle is reduced and therefore the fuel efficiency is been increased considerably and it gives very good results in various mechanical properties and produces fine surfaces over the parts of vehicles [11] [12].

The natural fiber is made of pectin, lignin, cellulose and hemicelluloses. Each type of natural fiber consists of various proportions of these components in percentage levels. Pectin function is to hold the fiber together and the lignin function is to absorb the water content in the fibers. The functions of cellulose and level of hemicellulose decide the strength and stiffness of the fibers respectively [10] [14] [15].

Industries such as packaging, automobile, and construction but natural fiber composite are also finding its way into sport, aerospace and in electronic industries, this is mainly due to their various advantages compared to synthetic fibers and conventional ones [9]. Natural fiber reinforced composites are best suited for many designs that demands weight reduction, increasing the life of the product, high tolerance precision engineering and simple in operation production methods.

Two or more materials combined together which results in better properties than those of the homogeneous components is termed as a composite material. The reinforcement layer in the composites gives additional strength, and stiffness, to make them harder, stronger. Generally, the reinforcement is in the form of fiber or in particulate form. The length-to-diameter ratio is called as the aspect ratio; increase the aspect ratio gives more strength to the fibers [15]. The mechanical property of natural fiber is increased by treating it with chemicals like alkali $(\mathrm{NaOH})$ [15] [22].

For two hours the fiber is rinsed with $\mathrm{NaOH}$ solution to remove the unwanted soluble. (Cellulose, Hemicellulose, pectin and lignin etc...)For removing the excess $\mathrm{NaOH}$ in the fibers it is thoroughly washed with water and dried with direct sunlight for two days. This treatment is the best suited for improving the tensile and flexural properties of the composite and also this is simplest and effective. [16] [22] [37]

In general, the smaller the diameter of the fiber, the higher its strength, but the cost increases when the diameter becomes smaller. In this experimental study, palm fiber (Palmyra spout)-flax-glass fiber reinforced hybrid composites are developed in unidirectional, bi-directional cross and their mechanical properties, such as tensile strength, flexural strength, and impact strength are evaluated. The various experiments show that during tensile loading, composites sustain greater 
loads as the angle between the fiber orientation (bi-directional cross) and the load direction increases [28].

\section{MATERIALS USED}

Palm Fiber

The palm fiber (Borassusflabellifer) is a natural fiber obtained from various portions of palm tree which is found in abundance in the southern parts of India. The palm fibers are composite material designed by nature, the fibers are basically a rigid, crystalline cellulose microfibril- reinforced amorphous lignin and/or with hemicellulosic matrix. Most plant fibers are composed of cellulose, hemicellulose, lignin, waxes and some water-soluble compounds [12]. Generally, this fiber contains $60-80 \%$ cellulose, 5-20\% lignin and upto $20 \%$ moisture. The fibers from various parts of the palm tree were extracted either by retting process or mechanical processing or hand processing. The fibers are soaked for two weeks and then it is washed with water and allow it dry completely in natural sunlight to obtain long and dried long uniform fibers [14]. The fibers are extracted from various parts of palm trees are Palm leaf stalk, Palm leaf sheath, Palm fruit, and Palm petioler respectively. Through research experimental, the palm fruit fiber has good mechanical properties [15] like specific strength, tensile strength, and hardness etc.

The palm fiber extracted from palm fruit gives more strength, the tensile behavior of the palm fibers increases with long sized fibers extracted from palm fruit[15]. Glass fiber and carbon fibers have more density than palm fiber ,therefore, the specific strength is better for the palm fiber compared with other fibers. Through the various experimental investigations, it has been found, the palm fiber especially the fiber from palm fruit gives more tensile strength and young's modulus compared with other natural fibers. The major compositions present in the palm fiber are cellulose, lignin and pectin. The palm fibers normally have more percentage of cellulose content which enables the fiber with better mechanical strength [16].

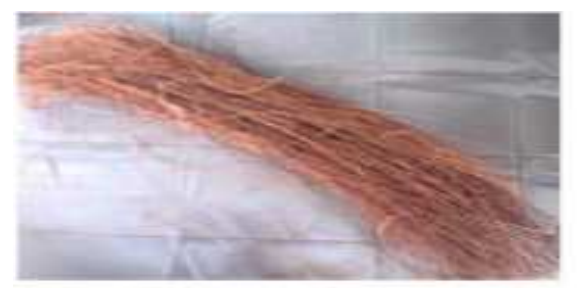

Pal m leaf stalk fiber

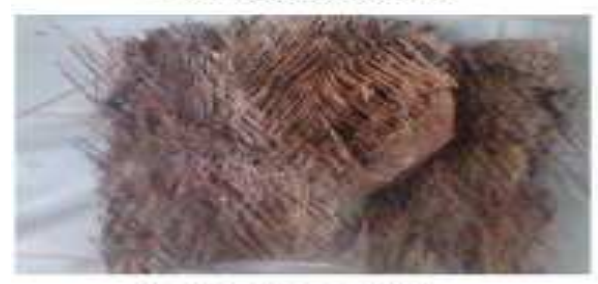

Palm leaf sheath fiber

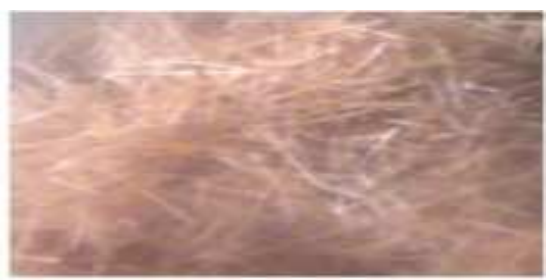

Palm fruit fiber

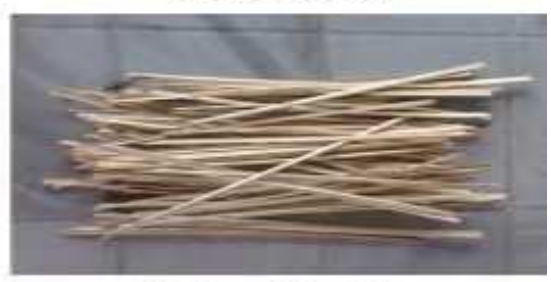

Palm petiole fiber

Figure 1: Extracted Fibers from Various Parts of Palm Tree

\section{Flax Fiber}

Flax fiber is extracted from the bast beneath the surface of the stem of the flax plant. Flax fiber is soft, lustrous, and flexible; bundles of fiber have the appearance of blonde hair, hence the description flaxen hair. Flax fiber is the strongest fiber in cellulosic fibers (plant fibers), two to three times stronger than cotton fiber, therefore it is more resistant to wear and abrasion [17]. Flax fiber is extracted from the skin of the flax stem plant. Flax has the best heat resistance and 
conductivity. Flax material has the potential of load-bearing constituents in composite materials due to their adjective properties such as high stiffness to weight ratio [18] [19].

\section{Glass Fiber}

Generally, polymers and carbon fiber have good mechanical properties but in recent evolution, this will be limited to some technological applications. The researchers have found the glass fibers which are extracted from glass having finely sized in the diametral range of 10-25 micron levels will be used in recent the development of composite materials. The glass fibers comparatively have more strength and rigidity and also the cost of glass fiber is low. Therefore in the recent development in NFC's these glass fibers are majorly used as a reinforcement material to manufacture lightweight and relatively strong composite materials. Lightweight fiber-reinforced polymer (FRP) composite material called glassreinforced plastic (GRP), also popularly known as "fiberglass" [20] [21].

\section{E-Glass Fiber}

In polymer matrix composites the E-glass is been used in a major number of investigations. The E-glass used in polymer composite is generally called as fiberglass and it has very well in mechanical and physical properties [23]. Through the recent experimental investigations, for some extent, only this E-glass will have good mechanical properties beyond that it fails and not be sufficient in some instances. Therefore the E-glass composition has been modified to produce more desirable properties [24]. A higher stiffness material resulting from this is S-Glass.

\section{S-Glass Fiber}

S-Glass found to have excellent fiber forming capabilities and is now used almost exclusively as the reinforcing phase in the material commonly known as fiberglass. S- Glass products are the ultimate in high strength glass fiber that provides the highest level of performance available in all glass fibers, Produced with a higher level of silica than standard glass fiber products, S-glass fibers offer the ultimate physical properties including high tensile and compressive strength (Fig.1), high-temperature resistance, and improved impact resistance [24].

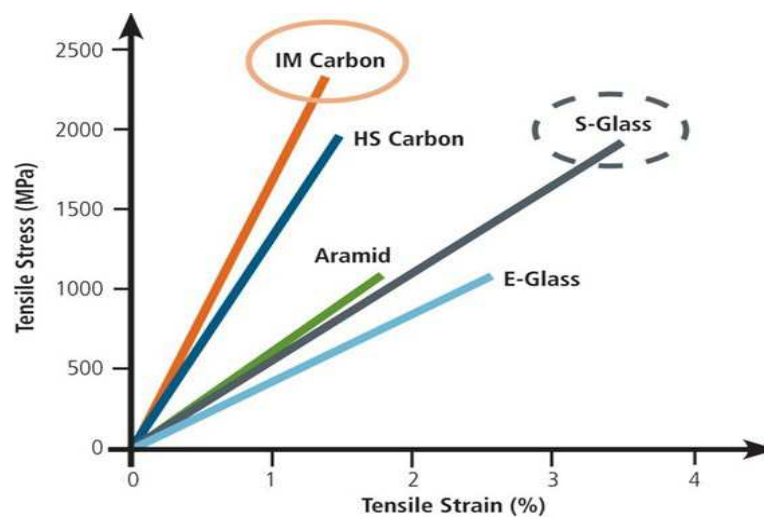

Figure 2: Tensile Properties of Various Types of Fibers

S-2 glass fibers are a magnesium alumino-silicate produced for textile substrates or reinforcements in composite applications. In hybrid composites, S-glass is added as the top and bottom layer to improve the tensile strength and surface finish. The required interfacial adhesion and bonding between various layers of fiber is achieved by using Epoxy resin (LY556) and Hardener (HY951) mixed in a ratio of 10:1 [25]. 


\section{MANUFACTURING METHODS OF NFCs}

\section{Hand Lay-Up Process}

The composite materials used for the present investigation is fabricated by hand layup process. In this work, Initially, the fiber mats of uniform thickness were prepared with flax and palm fibers. The composite laminate consists of multilayers. The mats were impregnated with epoxy resin then; the basalt fibers are dried under the hot sun to remove their moisture for more than 24 hours. Now, the fiber layers are washed in the acetone thinner before they are used for fabrication. Acetone removes the impurities and makes them ready for binding with the resin releasing agent is applied on the top of the table. The Flax fibers are spread on the base plate of the molding box which is placed on the table. It is then completely spread with epoxy resin and hardness mixture is applied on the flax fiber with brush and smoothens with a roller. Then, a layer of GFRP is placed on the resin-hardener mixer above which next layer of flax fiber is spread. Similarly, multiple layers are placed to prepare the composite (3 layerFlax/palm and 4 Layer GFRP). In this review, it is planned to prepare 3 types of composite. Type 1 composite contains palm fiber arranged inclined at $45^{\circ}$ to each other in all the 3 layers which resemble inclined orientation. Type 2 composite contains flax-fiber similar to type 1 . Type 3 contains both fibers. In each layers, both fibers are tilted at 45 degree in the subsequent layer (45degree orientation). In all the types, the top and bottom most layers is GFRP [25].

\section{MATERIAL PROPERTIES}

\section{Glass Fiber Mechanical Properties}

This glass fiber is mainly used to make the composite more strength and enhance the mechanical property also it produces a fine surface finish of the composite material. Therefore the base structure is made strong the entire composite polymers would be easily strengthened and improved [24]. The factors which are affecting the composite material performances are,

- The fiber orientation (the direction or angle in which the fibers are arranged)

- The fiber strength,

- The mechanical property of fibers, and

- The adhesion property [26] [27] [28].

The characteristics of natural fibers are very much important for determining the natural fiber composite material's mechanical property. The main characteristic of NFC's which decides the mechanical properties are fiber orientation, moisture absorption, and volume fraction. [25] [29] [30].

It clearly indicates that inclusion of glass fiber improves the load-bearing capacity and the ability to withstand bending of the composites. M. Ramesh et al. [7] investigated the mechanical properties of sisal, jute and glass fiber reinforced polyester composites. They observed that the addition of glass fiber into jute fiber composite resulted in maximum tensile strength [31] [32]. In the same way, they have observed that jute and sisal mixture composites sample is capable having maximum flexural strength and a maximum impact strength was obtained for the sisal fiber composite. The variation of tensile strength, flexural strength and compressive strength of epoxy based sisal-glass hybrid composites havebeen discussed in the papers [31] they have observed that $2 \mathrm{~cm}$ fiber length hybrid composites showed maximum optimal tensile, flexural and compressive strength than 1 and $3 \mathrm{~cm}$. The effect of alkali treatment on the tensile flexural and 
compressive properties has also been studied. It is found that treated hybrid composites showed higher strength than untreated composites [22].

Table 1: Mechanical Properties of Different Glass Fibers

\begin{tabular}{|l|l|l|l|l|c|c|}
\hline & E-Glass & R-Glass & HS2, HS4 & T-Glass & S-1 & S-2 \\
\hline Tensile Strength Gpa & $1.9-2.5$ & $3.1-3.4$ & $3.1-4.0$ & $4.0-4.2$ & $3.8-4.1$ & $4.3-4.6$ \\
\hline Tensile Modulus Gpa & $69-80$ & $86-89$ & $82-90$ & 84 & $85-87$ & $88-91$ \\
\hline
\end{tabular}

\section{Properties of Various Fibers}

Important properties of fibers are given below:

Table 2: Comparison of Typical Properties for Some Common Fibres

\begin{tabular}{|l|c|c|c|c|}
\hline \multirow{2}{*}{ Fiber } & \multicolumn{4}{|c|}{ Physical Properties } \\
\cline { 2 - 5 } & $\begin{array}{c}\text { Density } \\
\left(\mathbf{g} / \mathbf{c m}^{\mathbf{2}}\right)\end{array}$ & $\begin{array}{c}\text { Elongation } \\
(\mathbf{\%})\end{array}$ & $\begin{array}{c}\text { Tensile Strength } \\
(\mathbf{M p a})\end{array}$ & $\begin{array}{c}\text { Youngs Modulus } \\
(\mathbf{G p a})\end{array}$ \\
\hline Palm leaf & $1-1.2$ & $2-4.50$ & $97-196$ & $2.50-5.40$ \\
\hline Palm leaf sheath & $1.20-1.30$ & 2.84 & 220 & 4.8 \\
\hline Palm petiole & $0.7-1.55$ & 25 & 248 & 3.24 \\
\hline Palm fruit & 1.09 & 28 & 423 & $6-8$ \\
\hline Flax & 1.54 & $1-4$ & $345-2000$ & $27.5-85$ \\
\hline E-Glass & 2.55 & 2.5 & 2000 & 80 \\
\hline S-Glass & 2.46 & 2.8 & 4890 & 69 \\
\hline Kevlar 29 & 1.44 & 2.8 & 2860 & 136 \\
\hline Kevlar 49 & 1.44 & 4 & 3750 & \\
\hline
\end{tabular}

\section{RESULTS AND DISCUSSIONS}

A lot of research has been done on natural fiber reinforced polymer composites but research on palm-based polymer composites is very rare. Against this background, the present research work has been undertaken. Palmyra individual fiber is a better choice than other different fiber composites because it shows highest ultimate tensile stress under various tests performed. From the tables 1,2 it has been noted that the palm fruits and S-2 glass fiber have more strength and increased mechanical properties compared to other fibers respectively. This review deals with an objective to explore the potential of palm fiber and flax fiber composites reinforced with S-2 glass fiber for better mechanical properties mainly focused on strength and hardness of the composites. The experimental investigation on the composite lamina samples for various mechanical properties test in the future work. In future, there are tremendous investments on natural fibers, to bring an great impact on manufacturing sectors.

\section{CONCLUSIONS}

In recent day's lots of researchers progressed to improve the mechanical characteristics of natural fiber composites. The improvement is been made by fiber selection, extraction and treatment given to the fibers as well as processing of composite by proper selection of coatings over it. Comparing natural fiber and glass fiber reinforced composites found that natural fibers were superior in industrial application. Moreover, due to the usage of natural fibers in different engineering application and construction industries, it provides a way for economic development in rural areas. This paper reviewed the research that has mainly focussed on improving strength, stiffness and impact strength including the effect of moisture properties by treating it with chemical treatments. Through the experimental analysis by the various researchers, it has been found that, the moisture content in the fiber composites is been reduced phenomenally by the 
chemical treatments as discussed above. The major objective of the review paper is increasing the tensile strength of natural fiber composite by coating it with S2-glass fiber. Since S2-glass fiber have the adequate tensile strength and impact strength nature the mechanical properties of natural fiber composite (Palmyra-spout and flax fiber) reinforced with S2glass fiber is increased. Applications of NFCs have extended dramatically including load bearing and outdoor applications such as automotive exterior underfloor paneling, sports equipment and marine structures. Further, the experimental investigation to be done over the samples and through the results the application range, moisture resistance and fire retardance is been concluded. Overall the growth of NFC is growing rapidly and shown to be very positive future ahead of their application.

\section{ACKNOWLEDGEMENTS}

The authors would like to be obliged to Sri Sai Ram Engineering College for providing laboratory facilities and financial assistance under this research works.

\section{REFERENCES}

1. K. L. Pickering, M. G. AruanEfendy, T. M. Le, (2016) A review of recent developments in natural fibre composites and their mechanical performance. Part A 83 (2016) 98-112.

2. Malhotra, N., Sheikh, K. and Rani, S. (2012) A Review on Mechanical Characterization of Natural Fiber Reinforced Polymer Composites. Journal of Engineering Research and Studies, 3, 75-80.

3. K. P. Ashik, Ramesh S. Sharma. "A Review on Mechanical Properties of Natural Fibre Reinforced Hybrid Polymer Composites”, Journal of Minerals and Materials Characterization and Engineering, 2015, 3, 420-426.

4. Chandramohan, D. and Marimuthu, K. “A Review on Natural Fibers”(2011) IJRRAS, 8, 194-206.

5. U. S. Bongarde and V. D. Shinde, "Review on natural fiber reinforcement polymer composite," International Journal of Engineering Science and Innovative Technology, vol. 3, no. 2, pp. 431-436, 2014.

6. Dash, D., Samanta, S., Gautam, S. S. and Murlidhar, M. (2013) Mechanical Characterizations of Natural FiberReinforced Composite Materials. Advanced Materials Manufacturing \& Characterization, 3.

7. S. Shinoj, R. Visvanathan, S. Panigrahi, and M. Kochubabu, "Oil palm fiber (OPF) and its composites: a review," Industrial Crops and Products, vol. 33, no. 1, pp. 7-22, 2011.

8. Ramesh, M., Palanikumar, K. and Reddy, K. H. (2013) Mechanical Property Evaluation of Sisal-Jute-Glass Fiber Reinforced Polyester Composites. Composites: Part B, 48, 1-9.2012.12.04 K. P. Ashik, R. S. Sharma 426

9. Amit Rai et al Int. Journal of Engineering Research and Applications, Applications and Properties of Fibre Reinforced Concrete ISSN: 2248-9622, Vol. 4, Issue 5(Version 1), May 2014, pp.123-131

10. L. Mwaikambo, "Review of the history, properties and application of plant fibres," African Journal of Science and Technology, vol. 7, no. 2, p. 121, 2006.

11. Holbery, J., Houston, D., Natural-Fiber-Reinforced Polymer Composites in Automotive Applications, JOM, 2006, 11 , 80-86.

12. F. M. Al-Oqla and S. M. Sapuan, "Natural fiber reinforced polymer composites in industrial applications: feasibility of date palm fibers for sustainable automotive industry,” Journal of Cleaner Production, vol. 66, pp. 347-354, 2014.

13. C. Alves, P. M. C. Ferrão, A. J. Silva et al., "Ecodesign of automotive components making use of natural jute fiber composites," Journal of Cleaner Production, vol. 18, no. 4, pp. 313-327, 2010. 
14. Abu Bakar A. Hariharan, H. P. S. Abdul Khalil., "Lignocellulose-based Hybrid Bilayer Laminate Composite: Part I - Studies on Tensile and Impact Behavior of Oil Palm Fiber-Glass Fiber-reinforced Epoxy Resin," Volume: 39 issue: 8, page(s): 663684 Issue published: April 1, 2005.

15. M. A. NorulIzani, M. T. Paridah, U. M. K. Anwar, M. Y. Mohd Nor, and P. S. H'Ng, "Effects of fiber treatment on morphology, tensile and thermogravimetric analysis of oil palm empty fruit bunches fibers," Composites Part B: Engineering, vol. 45, no. 1, pp. 1251-1257, 2013.

16. AhmadAlawar, Ahmad M. Hamed, Khalifa Al-Kaabi., "Characterization of treated date palm tree fiber as composite reinforcement, "Volume 40, Issue 7, October 2009, Pages 601-606.

17. M Jacob, S Thomas, KT Varughese., "Mechanical properties of sisal/oil palm hybrid fiber reinforced natural rubber composites," Composites science and Technology-Elsevier., Volume 64, Issues 7-8, June 2004, Pages 955-965.

18. H. L. Bos, M. J. A. Van Den Oever, O. C. J. J. Peters "Tensile and compressive properties of flax fibres for natural fibre reinforced composites,” Journal of Materials Science, April 2002, Volume 37, Issue 8, pp 1683-1692.

19. C. Baley "Analysis of the flax fibres tensile behaviour and analysis of the tensile stiffness increase," Composites PartA:Applied Science and Manufacturing, Volume 33, Issue 7, 1 July 2002, Pages 939-948.

20. Daniel Wolf, Oliver Deisser, Raman Chaudhari, Holger Ahlborn "Glass Fibre Composite," System for Chassis ConceptsJuly 2012, Volume 1, Issue 7, pp 38-42.

21. PaulWambua, Jan ivens, IgnaasVerpoest "Natural fibres: can they replace glass in fibre reinforced plastics?," Composites Science and TechnologyVolume 63, Issue 9, July 2003, Pages 1259-1264.

22. Xue Li, Lope G. Tabil, Satyanarayan Panigrahi "Chemical Treatments of Natural Fiber for Use in Natural Fiber-Reinforced Composites: A Review," Journal of Polymers and the Environment January 2007, Volume 15, Issue 1, pp $25-33$.

23. G. Scarinci, G. Brusatin, LBarbieri, A. Corradi, I. Lancellotti, P. Colombo, S. Hreglich, R. Dall'Igna "Vitrification of industrial and natural wastes with production of glass fibres," Journal of the European Ceramic Society Volume 20, Issues 14-15, December 2000, Pages 2485-2490.

24. Jayabal, S., Natarajan, U. and Sathiyamurthy, S. (2011) Effect of Glass Hybridization and Stacking Sequence on Mechanical Behaviour of Interply Coir-Glass Hybrid Laminate. Bulletin of Materials Science, 34, 293-298.

25. J. Summerscales, S. Grove "7 - Manufacturing methods for natural fibre composites," Natural Fibre Composites Materials, Processes and Applications2014, Pages 176-215.

26. H. Ku, H. Wang, N. Pattarachaiyakoop, and M. Trada, "A review on the tensile properties of natural fiber reinforced polymer composites," Composites Part B: Engineering, vol. 42, no. 4, pp. 856-873, 2011.

27. F. Z. Arrakhiz, M. El Achaby, M. Malha et al., "Mechanical and thermal properties of natural fibers reinforced polymer composites:doum/low density polyethylene," Materials \& Design, vol. 43, pp. 200-205, 2013.

28. V. S. Srinivasan, S. R. Boopathy, D. Sangeetha, and B. V. Ramnath, "Evaluation of mechanical and thermal properties of banana-flax based natural fibre composite," Materials \& Design, vol. 60, pp. 620-627, 2014.

29. Samivel. P, A Ramesh Babu "Mechanical Behavior of Stacking Sequence in Kenaf and Bananafiber Reinforced-Polyester Laminate," ISSN 2278 - 0149 www.ijmerr.com Vol. 2, No. 4, October 2013 @ 2013 IJMERR.

30. J. Gassan and A. K. Bledzki, "Possibilities for improving the mechanical properties of jutelepoxy composites by alkali treatment of fibres," Composites Science and Technology, vol. 59, no. 9, pp. 1303-1309, 1999. 
31. M. Z. Rong, M. Q. Zhang, Y. Liu, G. C. Yang, and H. M. Zeng, "The effect of fiber treatment on the mechanical properties of unidirectional sisal-reinforced epoxy composites," Composites Science and Technology, vol. 61, no. 10, pp. 1437-1447, 2001.

32. Gopinath, A., Senthil Kumar, M. and Elayaperumal, A. (2014) Experimental Investigations on Mechanical Properties of Jute Fiber Reinforced Composites with Polyester and Epoxy Resin Matrices. 12th Global Congress on Manufacturing and Management, GCMM 2014, Procedia Engineering.

33. Alavudeen, A., Rajini, N., Karthikeyan, S., Thiruchitrambalam, M. and Venkateshwaren, N. (2015) Mechanical Properties of Banana/KenafFiber-Reinforced Hybrid Polyester Composites: Effect of Woven Fabric and Random Orientation. Materials and Design, 66, 246-257.

34. Sanjeevamurthy, G. C., Rangasrinivas, G. and Manu, S. (2012) Mechanical Performance of Natural Fiber-Reinforced EpoxyHybrid Composites. International Journal of Engineering Research and Applications (IJERA), 2, 615-619.

35. Zamri, M. H., Akil, H. M., Bakar, A. A., Ishak, Z. A. M. and Cheng, L. W. (2012) Effect of Water Absorption on Pultruded Jute/Glass Fiber-Reinforced Unsaturated Polyester Hybrid Composites. Journal of Composite Materials, 46, 51 - 61.

36. Gujjala, R., Ojha, S., Acharya, S. K. and Pal, S. K. (2013) "Mechanical Properties of Woven Jute-Glass Hybrid-Reinforced Epoxy Composite."Volume:48 issue: 28, page(s): 3445-3455 Journal of Composite Materials.

37. M. M. Kabir, H. Wang, K. T. Lau, and F. Cardona, "Chemical treatments on plant-based natural fibre reinforced polymer composites: an overview, " Composites Part B: Engineering, vol. 43, no. 7, pp. 2883-2892, 2012. 
